\title{
EVALUATION OF CBNAAT IN THE DIAGNOSIS OF SMEAR NEGATIVE TUBERCULOSIS IN A TERTIARY CARE HOSPITAL
}

\author{
Rachamadugu Hymavathi', Gundela Swarnalatha², Beldona Nagajyothi', Basireddy Srikanth Reddy ${ }^{4}$ \\ ${ }_{1}^{1}$ Assistant Professor, Department of Microbiology, Kurnool Medical College, Kurnool. \\ 2 Professor and HOD, Department of Microbiology, Kurnool Medical College, Kurnool. \\ ${ }^{3}$ Associate Professor, Department of Microbiology, Kurnool Medical College, Kurnool. \\ ${ }^{4}$ Assistant Professor, Department of Microbiology, Kurnool Medical College, Kurnool.
}

\begin{abstract}
BACKGROUND
ABSTRACT

Tuberculosis is world's one of the deadliest communicable diseases, which requires early diagnosis and treatment. As smear confirmation requires at least 10000 bacilli/mL and conventional culture methods take 4 - 8 weeks, nucleic acid amplification methods are better option for early diagnosis of the disease.
\end{abstract}

\section{MATERIALS AND METHODS}

In the present study, we evaluated the diagnostic yield of CBNAAT (Cartridge Based Nucleic Acid Amplification Technique) in the detection of Tuberculosis in smear negative patients attending Government General Hospital, Kurnool, Andhra Pradesh.

\section{RESULTS}

Out of the total 3157 suspected Tuberculosis cases 2850 were smear negative samples, out of which 353 samples were diagnosed as CBNAAT positive with 19 Rifampicin resistance cases.

\section{CONCLUSION}

From this study, NAA technique is found to be a better diagnostic method when compared to the microscopy.

\section{KEYWORDS}

CBNAAT, Fluorescent Microscopy, Sensitivity, Specificity.

HOW TO CITE THIS ARTICLE: Hymavathi R, Swarnalatha G, Nagajyothi B, et al. Evaluation of CBNAAT in the diagnosis of smear negative tuberculosis in a tertiary care hospital. J. Evolution Med. Dent. Sci. 2017;6(90):6273-6276, D0I: $10.14260 /$ jemds/2017/1364

\section{BACKGROUND}

Tuberculosis is an ancient and potentially fatal communicable disease caused mainly by M. tuberculosis complex (MTC), that can affect any part of the body with lungs being the most common organ involved. It is estimated that $1 / 3$ of the world's population (2 Billion) is infected with this

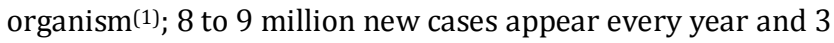
million persons die from this disease. India is one of the worst affected countries and having $>40 \%$ population infected, out of which 3 million are highly infectious open cases. Half million people die from this infection every year in India- one every minute, which reflects the magnitude of this highly fatal disease.(2)

If $\mathrm{TB}$ is to be eliminated as a global problem, earlier diagnosis and treatment will be essential. Tuberculosis can be diagnosed by Smear microscopy either by Ziehl-Neelsen's or Fluorescent staining; conventional culture either liquid or solid culture methods or molecular methods like GeneXpert, PCR and CBNAAT. Even though smear microscopy is easy and economical, it has some problems in detecting tuberculosis in patients with low bacterial load and differentiation is also

'Financial or Other Competing Interest': None.

Submission 23-10-2017, Peer Review 05-11-2017,

Acceptance 08-11-2017, Published 20-11-2017.

Corresponding Author:

Dr. Gundela Swarnalatha,

Professor and HOD,

Department of Microbiology,

KMC, Kurnool-518002.

E-mail: swarnalathagundela@gmail.com

DOI: $10.14260 /$ jemds/2017/1364

\section{(c) (i) $\$$}

difficult with other mycobacteria. Conventional culture will take 2 to 8 weeks to obtain the growth, which hampers the early diagnosis and treatment.(3,4) Drug resistant tuberculosis has become a problem in high TB countries including India.

Keeping in view of all the above problems, WHO has recently recommended a real time PCR test called CBNAAT as a primary aid for earlier and better diagnosis of the infection including Drug resistance.

\section{Aims and Objectives \\ 1. To evaluate the efficacy of CBNAAT in smear negative cases in the diagnosis of tuberculosis. \\ 2. To detect Rifampicin Resistance.}

\section{MATERIALS AND METHODS}

The present study was a prospective observational study conducted in the Department of Microbiology, Kurnool Medical College and Government General Hospital, Kurnool from January 2016 to July 2017. All the suspected tuberculosis patients attending GGH were selected, came out to be a total of 3157 . These cases were included irrespective of age and sex. The study group includes pulmonary and extrapulmonary samples including sputum, pleural fluid, CSF, pus, lymph nodes, paraspinal and gastric aspirates, ascitic and pericardial fluids and biopsy tissues.

\section{Sample Processing}

After the informed consent taken from the patients, samples were collected with suspected TB in a sterile container and transported to the laboratory within one hour. Samples were 
screened by microscopic examination (Ziehl-Neelsen's stain and fluorescent stain). All the smear negative cases were subjected to CBNAAT testing.

\section{CBNAAT}

Using the sterile pipette provided with the kit, sample was drawn from the container $1 \mathrm{~cm}$ past the minimum mark and transferred into the large opening at the bottom right of the cartridge slowly without shaking. After closing the lid, the program was started. Cartridge was put in the module after the initialisation and the module door was closed. After the program completed, the cartridge was removed and disposed off in the Biohazard Waste disposal bin and the results were recorded electronically.

\section{Statistical Analysis}

Data was entered in Excel version 2007. Data analysed in percentages and accuracy tests like sensitivity, specificity, false positives, false negatives, positive predictive value and negative predictive value were done. Results were recorded and interpreted in Bar charts and Pie diagrams.

\section{RESULTS}

A total of 3157 suspected tuberculosis patients were screened by microscopy. Out of them, 2850 were smear negative (Table 1 and Fig. 1). Out of these 2850 samples tested by CBNAAT 353 samples were detected positive by CBNAAT positive, 2389 cases as negative and 108 samples had errors (Table 2 and Fig. 2).

\begin{tabular}{|c|c|c|}
\hline Microscopy & $\begin{array}{c}\text { Number of } \\
\text { Samples }\end{array}$ & $\begin{array}{c}\text { Percentage } \\
\text { (\%) }\end{array}$ \\
\hline Smear Positive & 307 & 9.72 \\
\hline Smear Negative & 2850 & 90.27 \\
\hline Total Samples & $\mathbf{3 1 5 7}$ & $\mathbf{1 0 0}$ \\
\hline \multicolumn{2}{|c|}{ Table 1. Percentage of Smear Positive Cases } \\
\hline
\end{tabular}

\begin{tabular}{|c|c|c|}
\hline CBNAAT Positive & No. of Samples & (\%) \\
\hline CBNAAT Positive & 353 & 12.38 \\
\hline CBNAAT Negative & 2389 & 83.8 \\
\hline Errors (Invalid and No results) & 108 & 3.78 \\
\hline $\begin{array}{c}\text { Total Smear Negative } \\
\text { Samples }\end{array}$ & $\mathbf{2 8 5 0}$ & $\mathbf{1 0 0}$ \\
\hline \multicolumn{2}{|c|}{ Table 2. Percentage of CBNAAT Positive Cases } \\
\hline
\end{tabular}

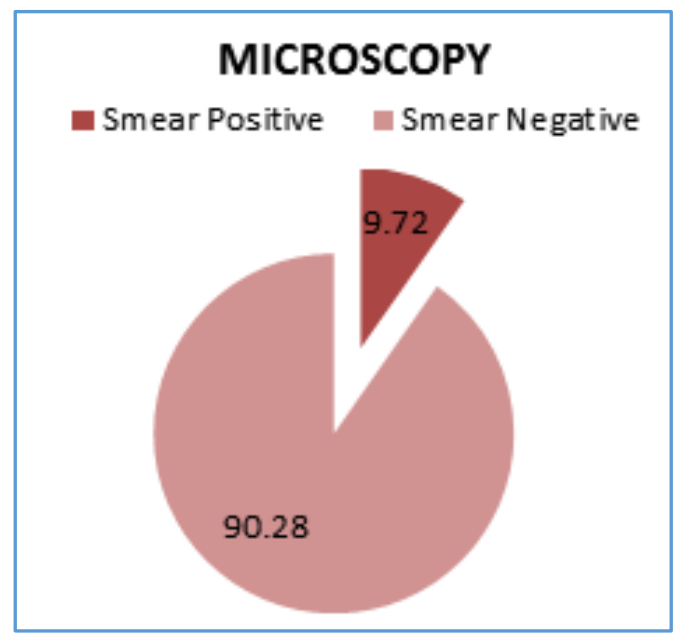

Figure 1. Microscopy Results

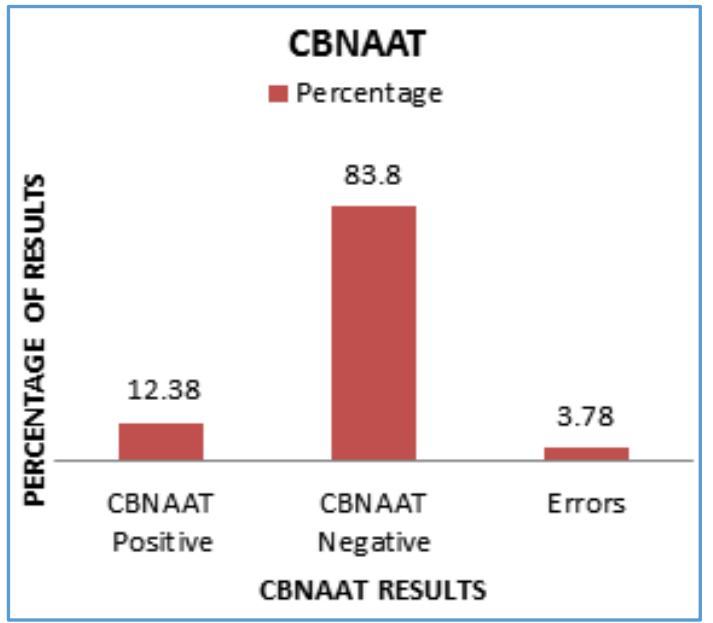

Figure 2. CBNAAT Results

Out of the 2850 smear negative cases, 2585 were pulmonary samples and 265 were extrapulmonary samples (Table 3 and Fig. 3).

\begin{tabular}{|c|c|c|}
\hline Sample & Number of Samples & (\%) \\
\hline Pulmonary Samples & 2585 & 90.7 \\
\hline Extrapulmonary Samples & 265 & 9.3 \\
\hline Total Smear Negatives & 2850 & 100 \\
\hline
\end{tabular}

\section{Table 3. Distribution of Pulmonary and Extrapulmonary} Samples

Out of 2585 pulmonary specimens screened, 335 (\%) were positive by CBNAAT, whereas 18 out of 265 extrapulmonary samples were identified as positive by CBNAAT (Table 4 and Fig. 4).

\begin{tabular}{|c|c|c|}
\hline Positive Sample Types & Number of Samples & (\%) \\
\hline Pulmonary Positive & 335 & 94.9 \\
\hline Extrapulmonary & 18 & 5.1 \\
\hline Total Positives & 353 & 100 \\
\hline Table 4. CBNAAT Positive Samples in Pulmonary and \\
Extrapulmonary Samples \\
\hline
\end{tabular}

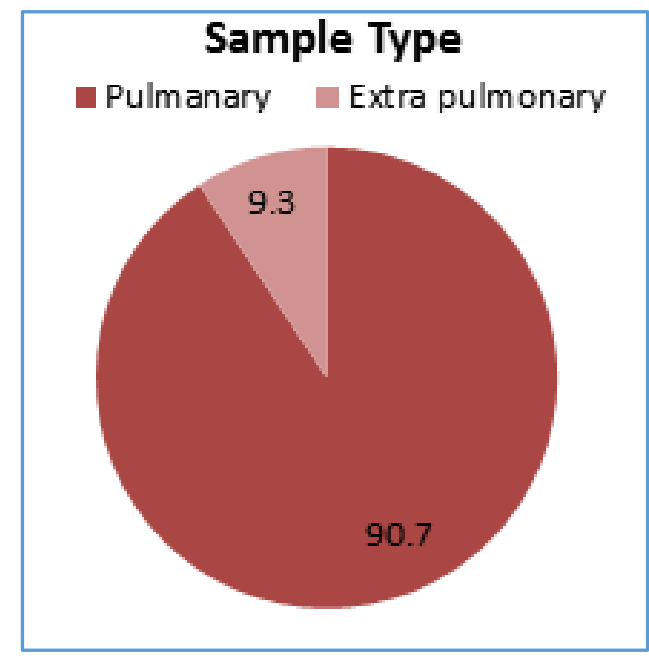

Figure 3. Distribution of Samples 


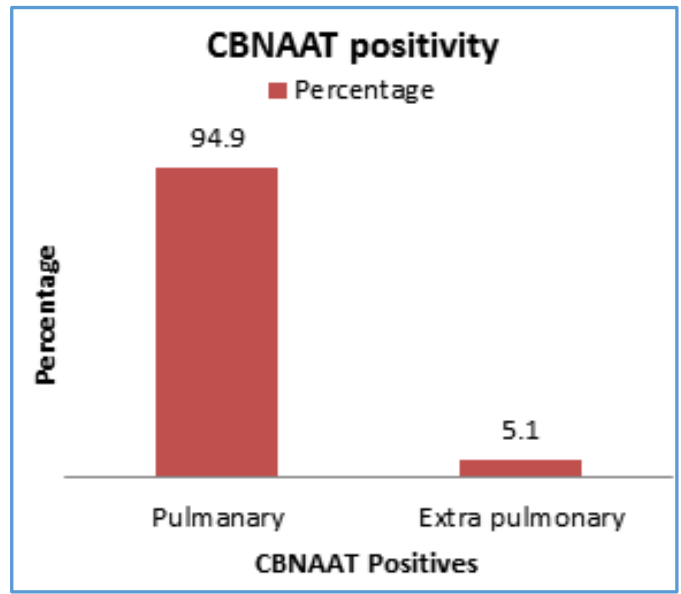

Figure 4. CBNAAT Positive Percentage

Out of 353 positives identified by CBNAAT 330 were sensitive to rifampicin, while MDR TB was detected in 19 samples (5.4\%); 4 samples have shown indeterminate results (Table 5 and Fig. 5, 6).

\begin{tabular}{|c|c|c|}
\hline Drug Resistance Pattern & No. of Samples & $(\%)$ \\
\hline Rifampicin Sensitive (RS) & 330 & 93.4 \\
\hline Rifampicin Resistant (RR) & 19 & 5.4 \\
\hline Rifampicin Indeterminate (RI) & 4 & 1.2 \\
\hline Total Positives & 353 & 100 \\
\hline
\end{tabular}

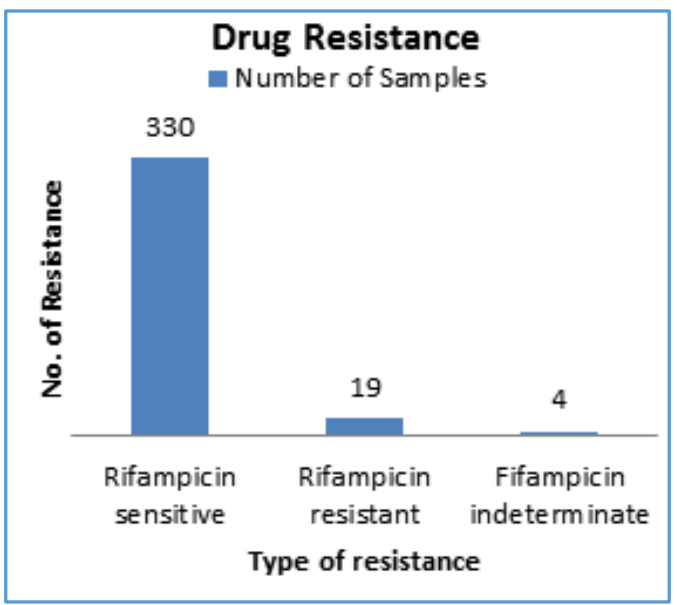

Figure 5. Drug Resistance

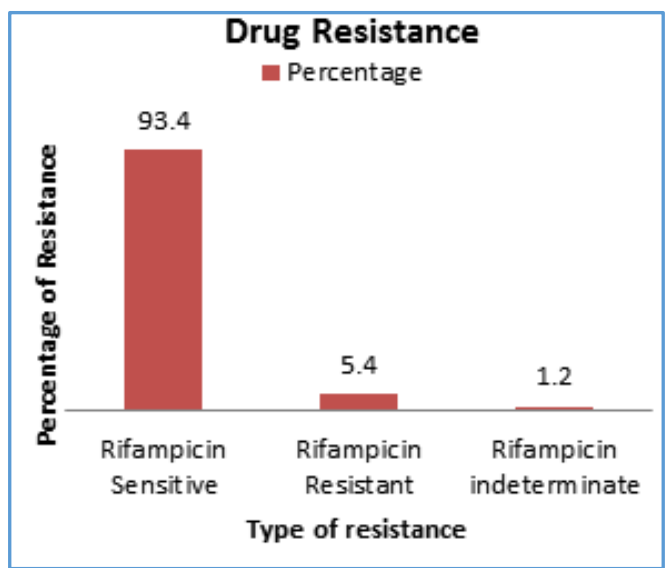

Figure 6. Percentage of Drug Resistance
HIV patients constituted 1252 out of the 2850 cases. Out of these 1252 HIV patients, 129 were identified positive by CBNAAT (Tables 6 and 7).

\begin{tabular}{|c|c|c|}
\hline Sample & Number of Samples & Percentage (\%) \\
\hline HIV Positive & 1252 & 43.9 \\
\hline HIV Negative & 1598 & 56.1 \\
\hline Total Samples & $\mathbf{2 8 5 0}$ & $\mathbf{1 0 0}$ \\
\hline \multicolumn{3}{|c|}{ Table 6. Distribution of HIV Cases } \\
\hline
\end{tabular}

\begin{tabular}{|c|c|c|}
\hline $\begin{array}{c}\text { CBNAAT Results in HIV } \\
\text { Positives }\end{array}$ & $\begin{array}{c}\text { Number of } \\
\text { Samples }\end{array}$ & $\begin{array}{c}\text { Percentage } \\
\text { (\%) }\end{array}$ \\
\hline CBNAAT Positive & 129 & 10.3 \\
\hline CBNAAT Negative & 1123 & 89.7 \\
\hline Total HIV Positive & $\mathbf{1 2 5 2}$ & $\mathbf{1 0 0}$ \\
\hline Table 7. Percentage of CBNAAT Positives in HIV Patients \\
\hline
\end{tabular}

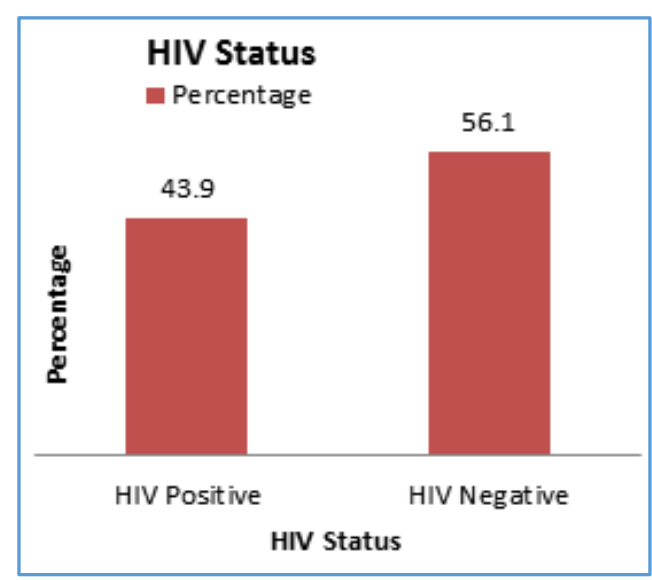

Figure 7. Distribution of HIV Status

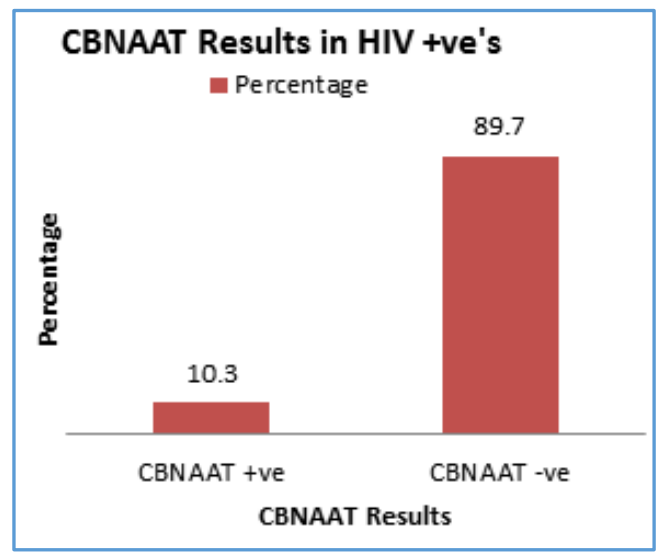

Figure 8. CBNAAT Results in HIV +ve's

\section{DISCUSSION}

Tuberculosis is one of the deadliest diseases ever known to the mankind. With the emergence of HIV, the incidence of TB has risen in the past few decades. Though there are many diagnostic tests available, the diagnosis is always a tedious procedure and has its limitations. Staining methods like ZN and fluorescent stains are rapid but lack the sensitivity, whereas culture is highly sensitive but is time consuming. Above this, there is emergence of drug resistance with MDR TB and XDR TB spreading rampantly in the community. Early diagnosis and prompt treatment is the motto for the tuberculosis control programs, which prevents the spread and emergence of drug resistance. In such a scenario, nucleic acid based diagnostic tests have emerged as a boon for the TB 
patients. With the introduction of CBNAAT, the diagnosis of TB has been eased. It not only helps in the early diagnosis of the patients, but also helps in identifying the rifampicin drug resistance within 2 hours which is extremely helpful for the categorical treatment of the patients.

In the present study the sensitivity, specificity, PPV and NPV of smear microscopy were $45.9 \%, 99.7 \%, 97.7 \%$ and 87.6\%; whereas the sensitivity, specificity, PPV and NPV of GeneXpert were $54 \%, 100 \%, 100 \%$ and $89.3 \%$. Our study results correlated well with the study results of many other researchers. In majority of the studies, microscopy sensitivity ranged from $22 \%$ to $41 \%$ and specificity from $95 \%$ to $100 \%$ which is similar to our study findings. $(5,6,7)$

In Agarwal et al(8) study for smear negative samples, the sensitivity and specificity of GeneXpert was $79.1 \%$ and 93.1\%. Many other studies have the sensitivities and specificities in the range of $57 \%-75 \%$ and $97 \%-100 \%$ respectively.(9-12)

In the present study rifampicin resistance was detected in $5.4 \%$ of the total cases, which is similar to the study conducted by Habte et $\mathrm{al}^{13}$ where the resistance rate was $4.2 \%$.

The present study highlights the importance of nucleic acid based testing methods, especially in smear negative cases. In extrapulmonary cases and HIV cases, it is often difficult to identify the pathogen by routine acid fast staining. In such cases, the GeneXpert plays pivotal role in the detection of the M. tuberculosis and also in the detection of the drug resistance to rifampicin.

\section{CONCLUSION}

Both conventional microscopy and GeneXpert are important tools in the rapid identification of tuberculosis. But as the sensitivity of the GeneXpert is higher than the microscopy and it also detects drug resistance to rifampicin, the utility of the CBNAAT is much more accountable especially in the smear negative tuberculosis cases. With extended coverage of GeneXpert services in both urban and rural level in the future will help not only in the early diagnosis and prompt treatment but also prevents the spread of infection and emergence of drug resistance.

\section{REFERENCES}

[1] World Health Organization. Global tuberculosis report 2014.

[2] Tuberculosis [Internet]. World Health Organization [cited 2015 may 1]. Available [2] from: http://www.who.int/mediacentre/factsheets/fs104/e $\mathrm{n} /$.
[3] Evans CA. GeneXpert-a game changer for tuberculosis control? PLoS Med 2011;8(7):e1001064.

[4] Centers for Disease Control and Prevention. Updated guidelines for the use of nucleic acid amplification tests in the diagnosis of tuberculosis. MMWR Morb Mortal Wkly Rep 2009;58(1):7-10.

[5] Le Palud P, Cattoir V, Malbruny B, et al. Retrospective observational study of diagnostic accuracy of the Xpert ${ }^{\circledR}$ MTB/RIF assay on fiberoptic bronchoscopy sampling for early diagnosis of smear-negative or sputum-scarce patients with suspected tuberculosis. BMC Pulm Med 2014;14:137.

[6] Barnard DA, Irusen EM, Bruwer JW, et al. The utility of Xpert MTB/RIF performed on bronchial washings obtained in patients with suspected pulmonary tuberculosis in a high prevalence setting. BMC Pulm Med 2015;15:103.

[7] Khalil KF, Butt T. Diagnostic yield of bronchoalveolar lavage gene Xpert in smear-negative and sputumscarce pulmonary tuberculosis. J Coll Physicians Surg Pak 2015;25(2):115-8.

[8] Agrawal M, Bajaj A, Bhatia V, et al. Comparative study of GeneXpert with ZN stain and culture in samples of suspected pulmonary tuberculosis. J Clin Diagn Res 2016;10(5):DC09-12.

[9] Norin J. A retrospective evaluation study of diagnostic accuracy of Xpert ${ }^{\circledR}$ MTB/RIF assay, used for detection of Mycobacterium tuberculosis in Greece. Örebro University, 2015.

[10] Zeka AN, Tasbakan S, Cavusoglu C. Evaluation of the genexpert MTB/RIF assay for rapid diagnosis of tuberculosis and detection of rifampin resistance in pulmonary and extrapulmonary specimens. J Clin Microbiol 2011;49(12):4138-41.

[11] Armand S, Vanhuls P, Delcroix G, et al. Comparison of the Xpert MTB/RIF test with an IS6110-TaqMan realtime PCR assay for direct detection of mycobacterium tuberculosis in respiratory and nonrespiratory specimens. J Clin Microbiol 2011;49(5):1772-6.

[12] Zmak L, Jankovic M, Jankovic VK. Evaluation of Xpert MTB/RIF assay for rapid molecular diagnosis of tuberculosis in a two-year period in Croatia. Int J Mycobacteriol 2013;2(3):179-82.

[13] Habte D, Melese M, Hiruy N, et al. The additional yield of GeneXpert MTB/RIF test in the diagnosis of pulmonary tuberculosis among household contacts of smear positive TB cases. Int J Infect Dis 2016;49:17984. 